
\title{
$\begin{array}{ll}\text { Research Square } & \text { They should not be considered conclusive, used to inform clinical practice, } \\ \text { or referenced by the media as validated information. }\end{array}$
}

\section{Individual and Combined Effect of Famine Exposure and Obesity Parameters on Dyslipidemia in Mid-Aged and Older Adult: A Population-Based Cross- Sectional Study}

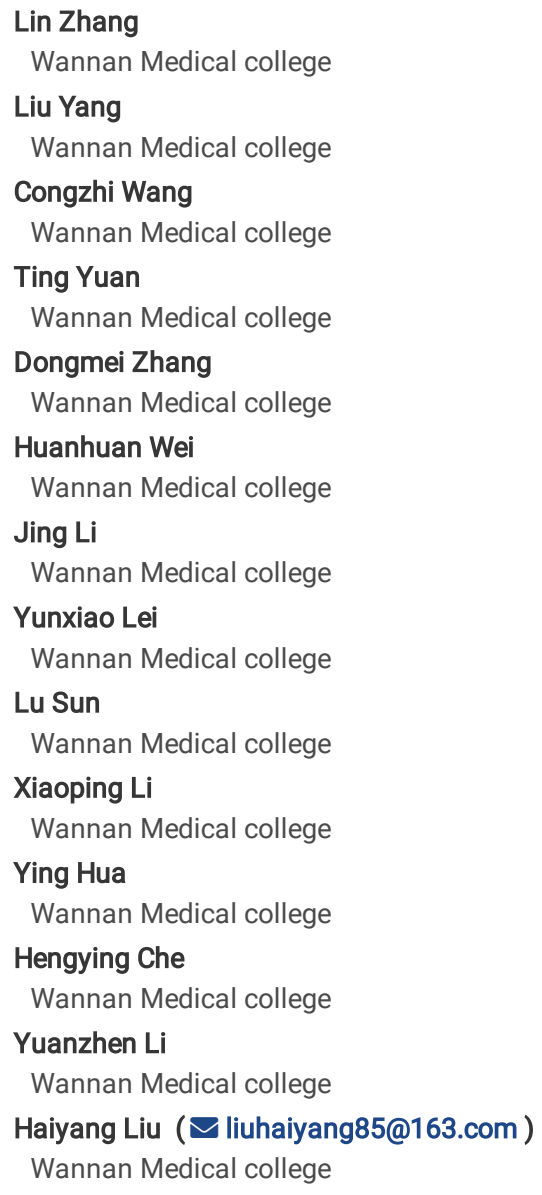

\section{Research Article}

Keywords: famine exposure, dyslipidemia, obesity parameters, body mass index, waist circle, the combined effect

Posted Date: September 24th, 2021

DOI: https://doi.org/10.21203/rs.3.rs-753103/v1

License: @ (i) This work is licensed under a Creative Commons Attribution 4.0 International License. Read Full License 


\section{Abstract}

Background: Undernutrition in early life may have a lifelong effect on adult health. The associations between undernutrition and obesity parameters and dyslipidemia were inconsistent. The present study aimed to investigate the individual and combined effects of famine exposure and obesity parameters on dyslipidemia in middle-aged and older Chinese.

Method: Data were selected from the China Health and Retirement Longitudinal Study Wave2011. The analytic sample included 9427 subjects aged 45 to 90. The present study analyzed data from 9427 middle-aged and older Chinese selected from the China Health and Retirement Longitudinal Study (CHARLS Wave2011). Differences between baseline characteristics and famine exposure/BMI levels/WC levels were evaluated using the Chi-square test, $\mathrm{t}$-test, and $\mathrm{F}$ test. Then, the difference in the prevalence of dyslipidemia between characteristic groups was also estimated by the Chi-square and t-test. Finally, multivariable-adjusted logistic regression models examined associations of famine exposure and obesity parameters with odds of prevalence of dyslipidemia.

Results: Among the 9427 participants, 1097(11.64\%) participants had been exposed to the Chinese famine during the fetal stage, whereas $3763(39.92 \%)$ participants and $3251(34.49 \%)$ participants had been exposed to the famine during childhood and adolescence/adult stage, respectively. Regarding the participants with BMI measurements,2771(29.39\%) were overweight and 1105(11.72\%) were obese, whereas 3955(41.95\%) of the participants with WC measurements were obese, respectively. Furthermore, 1899(43.23\%) reported having dyslipidemia in males and 1860(36.95\%) in females. In multivariableadjusted model, famine exposure and obesity parameters were associated with prevalence of dyslipidemia independently in total populations[(1) Model three c, famine exposure with prevalence of dyslipidemia: the fatal exposed vs no exposed group, 1.32 (95\% $\mathrm{Cl} 1.12,1.56)$; childhood-exposed vs no exposed group, 1.49 (95\% Cl 1.30, 1.70); the adolescence/adult-exposed vs no exposed group, $1.49(95 \% \mathrm{Cl} 1.30,1.71) ; P$ for trend=0.000; (2) Model three ${ }^{e}$, famine exposure with prevalence of dyslipidemia: the fatal exposed vs no exposed group, 1.29 (95\% Cl 1 .09, 1.52); childhood-exposed vs no exposed group, 1.39 ( $95 \% \mathrm{Cl} 1.22$, 1.59); the adolescence/adult-exposed vs no exposed group, $1.27(95 \% \mathrm{Cl} 1.11,1.46)$; $P$ for trend=0.002; (3) Model three ${ }^{g}$, BMI levels with prevalence of dyslipidemia: overweight vs normal, 2.06 (95\% Cl 1.86, 2.27); obesity vs normal, 2.82(95\% Cl 2.42, 3.27); $P$ for trend=0.000; (4) WC levels with prevalence of dyslipidemia: overweight vs normal, $2.24(95 \% \mathrm{Cl} 2.05,2.45)$ ]. When stratified by sex, the results in females were mostly similar to those in the total population. In a multivariable logistic regression model three ${ }^{c}$, associations between famine exposure and dyslipidemia were not observed [fatal exposed group vs nonexposed group: 0.98 (95\% Cl 0.75, 1.28); childhood-exposed group vs non-exposed group: 0.96 (95\% $\mathrm{Cl} 0.78,1.19)$; adolescence/adult exposed group vs nonexposed group: $0.86(95 \% \mathrm{Cl} 0.69,1.07)$ ] independently of BMI only ( $P$ for trend $=0.110)$. However, in a multivariable logistic regression model three $\mathrm{e}$, associations between famine exposure and dyslipidemia in male were partly observed [fatal exposed group vs non-exposed group: 0.97 ( $95 \% \mathrm{Cl} 0.74,1.26$ ); childhood-exposed group vs non-exposed group: 0.91 (95\% $\mathrm{Cl} 0.74,1.13)$; adolescence/adult exposed group vs non-exposed group: 0.73 (95\% $\mathrm{Cl} 0.59,0.91$ )] independently of BMI only ( $P$ for trend $=0.001)$. In general, the significant synergism between famine exposure and obesity parameters in lowering the prevalence of dyslipidemia was observed in males while the significant synergism in increasing prevalence of dyslipidemia was observed in females $(P$ interaction $=0.000$ ).

Conclusion: Individual and combined associations of obesity parameters and famine exposure with the prevalence of dyslipidemia were observed in middleaged and elderly Chinese.

\section{Background}

Dyslipidemia is characterized by an elevated low-density lipoprotein cholesterol (LDL-c), and/or triglycerides (TG), and/or total cholesterol (TC), and/or decreased high-density lipoprotein cholesterol (HDL-c). It was an important risk factor for cardiovascular disease (CVD), representing 31\%[1] of all global deaths. The prevalence of dyslipidemia was 34\%[2] among Chinese adults aged great than 18-years-old, and was $43 \%[3]$ among Chinese people aged 40 to 100 years. However, the rates of awareness, treatment, and control were $31.0 \%, 19.5 \%$, and $8.9 \%$, respectively. The prevalence of dyslipidemia among Chinese adults was high but awareness, treatment, and control of dyslipidemia were still low. Dyslipidemia is a serious public health problem and causes a serious burden to families and society. Therefore, it emphasizes the effective, practical, and sustainable promotion of prevention and treatment strategies to prevent dyslipidemia risk factors[4-6]. Though the etiology of dyslipidemia is complex, it was known as one of the strongest risk factors was overweight/obesity. Thus, increased body mass index (BMI) or centrally located body fat (especially waist circle [WC]) increases the risk of developing dyslipidemia. In addition to known and probable risk factors for dyslipidemia, early life mal-nutrition may also affect dyslipidemia.

It was hypothesized that early developmental adaption in response to malnutrition in early life, which are key determinants of short-term survival, have adverse metabolic outcomes[7-9]. Historical famine exposure has provided a unique and natural opportunity to test the hypothesis. From 1959-1961, China suffered from extreme food shortages. It was one of the most famine disasters in human history, 15-43 million people died. We selected participants from the age group for our study to understand the impact of malnutrition in early life on their health. Though some studies have indicated malnutrition in early life is relationship with the risk of developing chronic diseases in adult(e.g. type 2 diabetes[10], metabolic syndrome[11, 12], cardiovascular disease, hypertension[13, 14], and cognitive decline[15, 16]). Several famine studies[17-19] also have provided evidences to support the association between famine exposure and increased risk of dyslipidemia. However, it is not completed understood association and interaction analysis between famine exposure and obesity parameter and dyslipidemia in the older adult.

Therefore, in present study, we use the data derived from the China Health and Retirement Longitudinal Study (CHARLS) Wave1 to investigate the individual and combined effects of famine exposure and obesity parameter (BMI and WC) on dyslipidemia after adjustment for confounding variables.

\section{Methods}

\section{Study design and setting}


Data from the China Health and Retirement Longitudinal Study (CHARLS) Wave1 were used in our research.

The CHARLS was a nationally representative longitudinal study conducted by the China Centre for Economic Research at Peking University[20] from 2011. In the 2011 CHARLS Wave1, at baseline, 13107 individuals were recruited for a longitudinal study, after excluding participants who had missing values of the baseline characteristics and measurements, 9427 were included in the study. All data are openly published as microdata at http://charls.pku.edu.cn/index/zh$\mathrm{cn}$.html with no direct contact with all participants. The Ethics Committee of the China Centre for Economic Research at Peking University approved this study, all participants have provided the informed consent before the data collection.

\section{Subjects}

The subjects of the study were selected from the China Health and Retirement Longitudinal Study (CHARLS), Wave 1 (2011) [20]. The age of CHARLS involved 9427 individuals were [mean \pm standard deviation age $=59.51 \pm 9.32$ years, ranged from 45 to 90 years]. The mean and standard deviation of age were 60.26 \pm 9.23 years (ranged from 45 to 90 years) in males and $58.85 \pm 9.35$ years (ranged from 45 to 96 years) in females.

\section{Baseline Characteristics}

Baseline variables including age, $\operatorname{sex}(0=$ male, $1=$ female $)$, educational levels $(0=$ Illiterate, $1=$ Less than elementary school, $2=$ High school, $3=$ Above vocational school), marital status $(0=$ Single, $1=$ Married $)$, place of residence $(0=$ Rural, $1=$ Urban $)$, smoking habits $(0=$ No, $1=$ Former $\operatorname{smoke}, 2=$ Current smoke), alcohol consumption ( $0=$ No, $1=$ Less than once a month, $2=$ More than once a month), eating meals $(0=\leq 2$ meals per day, $1=3$ meals per day, $2=$ $\geq 4$ meals per day), social and leisure activities $(0=$ No, $1=$ Yes $)$, experience of a traumatic event $(0=$ No, $1=$ Yes $)$, physical exercise habit $(0=$ No, $1=$ Less than regular physical exercises, 2 = Regular physical exercises) were collected using a type of self-report method. Most variables were depending on our previous research studies[21-26].

\section{Measurements}

BMI was calculated based on the measured weight and height of the participants. Tapeline was localized at navel levels to read the waist circle (WC) at the end of exhalation. Using the standard China definition, BMI was categorized into three groups[27]: underweight and normal (BMI $\left.<24 \mathrm{~kg} / \mathrm{m}^{2}\right)$, overweight $(24$ $\left.\leq \mathrm{BMI}<28 \mathrm{~kg} / \mathrm{m}^{2}\right)$, and obesity $\left(\mathrm{BMI} \geq 28 \mathrm{~kg} / \mathrm{m}^{2}\right)$. Central obesity was defined as a WC[28] of $\geq 90 \mathrm{~cm}$ for men and $\geq 85 \mathrm{~cm}$ for women. Frozen plasma LDL-C, HDL-C, TG, and total TC from venous blood samples stored frozen at - $20 \circ \mathrm{C}$ were analyzed by the Youanmen Center for Clinical Laboratory at Capital Medical University using the enzymatic colormetric test. The dyslipidemia was defined based on the guidelines provided in the third report of the National Cholesterol Education Programme (NCEP) Adult Treatment Panel III (NCEP ATP III): TC $\geq 5.2 \mathrm{mmol} / \mathrm{L} ; \mathrm{TG} \geq 1.7 \mathrm{mmol} / \mathrm{L} ; \mathrm{HDL}-\mathrm{C}<1.0 \mathrm{mmol} / \mathrm{L}$ in male and $<1.3 \mathrm{mmol} / \mathrm{L}$ in female; LDL-C $\geq 3.4 \mathrm{mmol} / \mathrm{L}[29]$.

\section{Exposure Age And Exposed Stages}

Famine exposure is set up on the birth year. Like the previous Chinese famine study[30], participants were categorized into four exposure cohorts: no-exposed stage (born between 1963/01/01 and 1966/12/31), fetal exposed stage (born between 1959/01/01 and 1962/12/31), childhood exposed stage (born between 1949/01/01 and 1958/12/31), adolescence/adult exposed stage (born between1921/01/01 and 1948/12/31).

\section{Statistical analysis}

The data are presented as means and standard deviation (SD) unless indicated otherwise. Means and standard deviation (continuous data) were used to describe continuous variable (age), and number and percentage (categorical data) were used to assess the categorical variables (sex, educational levels, marital status, residence places, alcohol drinking, smoking habit, eating meals, social and leisure activities, the experience of a traumatic event, taking physical activity or exercise, famine exposure, BMI categories, WC categories, and dyslipidemia categories). Between-group differences according to dyslipidemia (dyslipidemia, no-dyslipidemia) were evaluated by the chi-square test (categorical data). Differences between baseline characteristics (marital status, residence places, social and leisure activities, the experience of a traumatic event, taking physical activity or exercise) and categories of famine exposure stages/BMI/WC were also evaluated using the chi-square test (categorical data). Age between groups was used by t-test or F-test. For the present study, logistic regression models were used to compute ORs with accompanying 95\% Cls as estimates of associations of BMI/WC categories and exposure stages separately and in combination, with the prevalence of dyslipidemia. Analyses were performed using SPSS software, version 25.0(IBM SPSS, Armonk, NY, USA), with a $5 \%$ significance level.

\section{Results}

Table 1 shows the basic characteristics of participants. A total of 9427 individuals were enrolled into the study, 4393(46.60\%) participants and 5034(53.40\%) participants were male and female, respectively. Among males, $461(10.49 \%)$ participants had been exposed to the Chinese famine during the fetal stage, whereas $1766(40.20 \%)$ participants and 1662(37.83\%) participants had been exposed to the famine during childhood and adolescence/adult stage, respectively. Among females, 636(12.63\%) participants had been exposed to the Chinese famine during the fetal stage, whereas $1997(35.70 \%)$ participants and $1589(31.57 \%)$ participants had been exposed to the famine during childhood and adolescence/adult stage, respectively. The distribution of history of accidental injury did not demonstrate significantly statistical difference among the four birth cohorts. On the other hand, the difference was observed in the 
distribution of age, sex, educational levels, place of residence, marital status, cigarette smoking, alcohol habit, eating habit, social events, and physical exercises habit. Regarding the males, 2879(65.95\%) were underweight and normal, $1127(25.65 \%)$ were overweight and $369(8.40 \%)$ were obese, whereas $2654(52.72 \%), 1644(32.66 \%)$ and $736(14.62 \%)$ of the females were underweight and normal, overweight, and obese, respectively. Furthermore, significant differences in distribution were observed between BMI levels in all of the variables, including age, sex, educational levels, marital status, place of residence, cigarette smoking, alcohol habit, eating habit, social events, and physical exercises habit, except for history of accidental injury. Among the WC measures, $1316(29.96 \%)$ were central obesity in males and $2639(52.42 \%)$ in females. The proportions on the characteristics were statistically different between the WC groups except for marital status. 
Table 1

Characteristics of participants in the cohort study by level of famine exposure, BMI, and central obesit:

\begin{tabular}{|c|c|c|c|c|c|c|c|c|c|c|}
\hline \multirow[t]{2}{*}{ Characteristics } & \multicolumn{6}{|c|}{ Famine exposure } & \multicolumn{4}{|l|}{ BMI } \\
\hline & No-exposed & $\begin{array}{l}\text { Fetal } \\
\text { exposed }\end{array}$ & $\begin{array}{l}\text { Childhood- } \\
\text { exposed }\end{array}$ & $\begin{array}{l}\text { Adolescence/ } \\
\text { adult- } \\
\text { exposed }\end{array}$ & $\chi 2 / F$ & $P$ & Normal & Overweight & Obesity & $\times 2$ \\
\hline $\mathrm{N}$ & 1316 & 1097 & 3763 & 3251 & & & 5551 & 2771 & 1105 & \\
\hline Age & $46.75 \pm 1.08$ & $50.27 \pm 1.17$ & $57.57 \pm 2.78$ & $70.02 \pm 5.71$ & 15498.488 & 0.000 & $60.66 \pm 9.60$ & $58.10 \pm 8.73$ & $57.26 \pm 8.40$ & 10\{ \\
\hline \multicolumn{11}{|l|}{ Gender } \\
\hline male & $504(38.30)$ & $461(42.02)$ & 1766(46.93) & 1662(51.12) & 72.572 & 0.000 & 2897(52.19) & $1127(40.67)$ & 369(33.39) & 181 \\
\hline female & $812(61.70)$ & 636(57.98) & 1997(53.07) & $1589(48.88)$ & & & 2654(47.81) & 1644(59.33) & $736(66.61)$ & \\
\hline \multicolumn{11}{|l|}{ Education } \\
\hline Illiterate & 147(11.17) & 192(17.5) & $1090(28.97)$ & 1284(39.50) & 792.449 & 0.000 & 1696(30.55) & $718(25.91)$ & $299(27.06)$ & 46. \\
\hline $\begin{array}{l}\text { Less than } \\
\text { elementary } \\
\text { school }\end{array}$ & $993(75.46)$ & $665(60.62)$ & $2336(62.08)$ & $1793(55.15)$ & & & $3388(61.03)$ & 1717(61.96) & 682(61.72) & \\
\hline High school & $124(9.42)$ & $204(18.60)$ & $255(6.78)$ & $44(1.35)$ & & & $326(5.87)$ & $217(7.83)$ & $84(7.60)$ & \\
\hline $\begin{array}{l}\text { Above } \\
\text { vocational } \\
\text { school }\end{array}$ & $52(3.95)$ & $36(3.28)$ & $82(2.18)$ & $130(4.00)$ & & & $141(2.54)$ & $119(4.29)$ & $40(3.62)$ & \\
\hline \multicolumn{11}{|l|}{ Marital status } \\
\hline Single & $48(3.65)$ & $55(5.01)$ & $303(8.05)$ & $762(23.44)$ & 578.51 & 0.000 & $813(14.65)$ & $251(9.06)$ & $104(9.41)$ & 63. \\
\hline Married & 1268(96.35) & 1042(94.99) & $3460(91.95)$ & $2489(76.56)$ & & & 4738(85.35) & $2520(90.94)$ & 1001(90.59) & \\
\hline \multicolumn{11}{|l|}{$\begin{array}{l}\text { Place of } \\
\text { residence }\end{array}$} \\
\hline Rural & $824(62.61)$ & 706(64.36) & 2413(64.12) & 2165(66.59) & 8.195 & 0.042 & $3894(70.15)$ & 1617(58.35) & $597(54.03)$ & 17 \\
\hline Urban & 492(37.39) & $391(35.64)$ & 1350(35.88) & 1086(33.41) & & & 1657(29.85) & 1154(41.65) & $508(45.97)$ & \\
\hline \multicolumn{11}{|l|}{$\begin{array}{l}\text { Cigarette } \\
\text { smoking }\end{array}$} \\
\hline NO & $914(69.45)$ & $700(63.81)$ & 2239(59.5) & 1854(57.03) & 123.017 & 0.000 & $3042(54.8)$ & 1856(66.98) & $809(73.21)$ & 26 \\
\hline Former smoke & $70(5.32)$ & $57(5.20)$ & $321(8.53)$ & 406(12.49) & & & $472(8.5)$ & 279(10.07) & 103(9.32) & \\
\hline Current smoke & $332(25.23)$ & $340(30.99)$ & 1203(31.97) & $991(30.48)$ & & & 2037(36.7) & $636(22.95)$ & 193(17.47) & \\
\hline \multicolumn{11}{|l|}{ Alcohol habit } \\
\hline NO & $880(66.87)$ & $712(64.9)$ & 2487(66.09) & $2262(69.58)$ & 24.565 & 0.000 & $3565(64.22)$ & 1944(70.16) & $832(75.29)$ & 73. \\
\hline $\begin{array}{l}\text { Less than } \\
\text { once a month }\end{array}$ & 117(8.89) & $110(10.03)$ & $306(8.13)$ & $207(6.37)$ & & & $448(8.07)$ & $209(7.54)$ & $83(7.51)$ & \\
\hline $\begin{array}{l}\text { More than } \\
\text { once a month }\end{array}$ & $319(24.24)$ & $275(25.07)$ & $970(25.78)$ & $782(24.05)$ & & & 1538(27.71) & 618(22.30) & 190(17.19) & \\
\hline \multicolumn{11}{|l|}{ Eating habit } \\
\hline $\begin{array}{l}\leq 2 \text { meals per } \\
\text { day }\end{array}$ & 193(14.67) & $149(13.58)$ & $439(11.67)$ & $465(14.3)$ & 22.098 & 0.001 & $837(15.08)$ & 298(10.75) & 111(10.05) & 57. \\
\hline $\begin{array}{l}3 \text { meals per } \\
\text { day }\end{array}$ & 1113(84.57) & $938(85.51)$ & $3259(86.61)$ & 2744(84.4) & & & $4620(83.23)$ & 2445(88.24) & $989(89.50)$ & \\
\hline $\begin{array}{l}\geq 4 \text { meals per } \\
\text { day }\end{array}$ & $10(0.76)$ & $10(0.91)$ & $65(1.73)$ & $42(1.29)$ & & & $94(1.69)$ & $28(1.01)$ & $5(0.45)$ & \\
\hline \multicolumn{11}{|l|}{ Social events } \\
\hline No & $565(42.93)$ & 465(42.39) & 1906(50.65) & 1732(53.28) & 65.433 & 0.000 & $2925(52.69)$ & $1269(45.80)$ & $474(42.90)$ & 57. \\
\hline Yes & 751(57.07) & $632(57.61)$ & 1857(49.35) & 1519(46.72) & & & $2626(47.31)$ & $1502(54.20)$ & $631(57.10)$ & \\
\hline
\end{tabular}




\begin{tabular}{|c|c|c|c|c|c|c|c|c|c|c|}
\hline \multirow[t]{2}{*}{ Characteristics } & \multicolumn{6}{|c|}{ Famine exposure } & \multicolumn{4}{|l|}{ BMI } \\
\hline & No-exposed & $\begin{array}{l}\text { Fetal } \\
\text { exposed }\end{array}$ & $\begin{array}{l}\text { Childhood- } \\
\text { exposed }\end{array}$ & $\begin{array}{l}\text { Adolescence/ } \\
\text { adult- } \\
\text { exposed }\end{array}$ & $X 2 / F$ & $P$ & Normal & Overweight & Obesity & $x^{2}$ \\
\hline No & 1191(90.5) & $986(89.88)$ & $3381(89.85)$ & 2949(90.71) & 1.734 & 0.629 & 4991(89.91) & 2497(90.11) & 1019(92.22) & 5.6 \\
\hline Yes & $125(9.5)$ & 111(10.12) & $382(10.15)$ & $302(9.29)$ & & & $560(10.09)$ & 274(9.89) & $86(7.78)$ & \\
\hline \multicolumn{11}{|l|}{$\begin{array}{l}\text { Physical } \\
\text { exercises habit }\end{array}$} \\
\hline $\begin{array}{l}\text { No physical } \\
\text { exercise }\end{array}$ & 794(60.33) & 644(58.71) & $2286(60.75)$ & $2080(63.98)$ & 16.153 & 0.013 & $3451(62.17)$ & $1658(59.83)$ & $695(62.90)$ & 14. \\
\hline $\begin{array}{l}\text { Less than } \\
\text { regular } \\
\text { physical } \\
\text { exercises }\end{array}$ & $258(19.6)$ & $234(21.33)$ & 744(19.77) & $561(17.26)$ & & & 1081(19.47) & 536(19.34) & $180(16.29)$ & \\
\hline $\begin{array}{l}\text { Regular } \\
\text { physical } \\
\text { exercises }\end{array}$ & $264(20.06)$ & 219(19.96) & 733(19.48) & $610(18.76)$ & & & 1019(18.36) & $577(20.82)$ & $230(20.81)$ & \\
\hline
\end{tabular}

Table 2 shows the characteristics of study participants categorized by dyslipidemia. Of the participants, 1899(43.23\%) reported having dyslipidemia in male and $1860(36.95 \%)$ in the female. Significant differences were observed in sex, place of residence, cigarette smoking, alcohol habit, social events, famine exposure, BMI levels, and WC groups $(P<0.05)$ between participants with and without dyslipidemia. 
Table 2

Characteristics of study participants of cross-sectional study categorized by dyslipidemia $(N=9427)$

\begin{tabular}{|c|c|c|c|c|c|}
\hline Variables & $\begin{array}{l}\text { Without dyslipidemia } \\
N=3759\end{array}$ & $\begin{array}{l}\text { Dyslipidemia } \\
\mathrm{N}=5668\end{array}$ & Total & $\chi 2 / \mathrm{t}$ & $P$ \\
\hline Age & $59.38 \pm 9.52$ & $59.59 \pm 9.19$ & $59.51 \pm 9.32$ & -1.085 & 0.278 \\
\hline \multicolumn{6}{|l|}{ Gender } \\
\hline male & 1899(50.52) & $2494(44.00)$ & $4393(46.60)$ & 38.578 & 0.035 \\
\hline female & $1860(49.48)$ & $3174(56.00)$ & $5034(53.40)$ & & \\
\hline \multicolumn{6}{|l|}{ Education } \\
\hline Illiterate & 1041(27.69) & $1672(29.50)$ & $2713(28.78)$ & 4.584 & 0.205 \\
\hline Less than elementary school & $2356(62.68)$ & $3431(60.53)$ & $5787(61.39)$ & & \\
\hline High school & $243(6.46)$ & $384(6.77)$ & $627(6.65)$ & & \\
\hline Above vocational school & 119(3.17) & 181(3.19) & $300(3.18)$ & & \\
\hline \multicolumn{6}{|l|}{ Marital status } \\
\hline Single & $452(12.02)$ & $716(12.63)$ & 1168(12.39) & 0.769 & 0.38 \\
\hline Married & $3307(87.98)$ & 4952(87.37) & $8259(87.61)$ & & \\
\hline \multicolumn{6}{|l|}{ Place of residence } \\
\hline Rural & 2562(68.16) & $3546(62.56)$ & $6108(64.79)$ & 31.011 & 0.000 \\
\hline Urban & 1197(31.84) & $2122(37.44)$ & $3319(35.21)$ & & \\
\hline \multicolumn{6}{|l|}{ Cigarette smoking } \\
\hline NO & 2177(57.91) & $3530(62.28)$ & $5707(60.54)$ & 42.537 & 0.000 \\
\hline Former smoke & $301(8.01)$ & $553(9.76)$ & $854(9.06)$ & & \\
\hline Current smoke & 1281(34.08) & $1585(27.96)$ & $2866(30.40)$ & & \\
\hline \multicolumn{6}{|l|}{ Alcohol habit } \\
\hline NO & $2453(65.26)$ & $3888(68.60)$ & $6341(67.26)$ & 11.897 & 0.003 \\
\hline Less than once a month & $321(8.54)$ & $419(7.39)$ & $740(7.85)$ & & \\
\hline More than once a month & $985(26.2)$ & 1361(24.01) & $2346(24.89)$ & & \\
\hline \multicolumn{6}{|l|}{ Eating habit } \\
\hline$\leq 2$ meals per day & $527(14.02)$ & $719(12.69)$ & $1246(13.22)$ & 3.51 & 0.173 \\
\hline 3 meals per day & $3182(84.65)$ & $4872(85.96)$ & $8054(85.44)$ & & \\
\hline$\geq 4$ meals per day & $50(1.33)$ & $77(1.36)$ & $127(1.35)$ & & \\
\hline \multicolumn{6}{|l|}{ Social events } \\
\hline No & 1965(52.27) & 2703(47.69) & $4668(49.52)$ & 19.013 & 0.000 \\
\hline Yes & 1794(47.73) & 2965(52.31) & $4759(50.48)$ & & \\
\hline \multicolumn{6}{|l|}{ History of accidental injury } \\
\hline No & 3398(90.4) & $5109(90.14)$ & $8507(90.24)$ & 0.172 & 0.678 \\
\hline Yes & $361(9.6)$ & $559(9.86)$ & $920(9.76)$ & & \\
\hline \multicolumn{6}{|l|}{ Physical exercises habit } \\
\hline No physical exercise & $2266(60.28)$ & $3538(62.42)$ & $5804(61.57)$ & 5.775 & 0.056 \\
\hline Less than regular physical exercises & $758(20.16)$ & 1039(18.33) & 1797(19.06) & & \\
\hline Regular physical exercises & 735(19.55) & 1091(19.25) & 1826(19.37) & & \\
\hline \multicolumn{6}{|l|}{ Famine exposure } \\
\hline No-exposed & $594(15.80)$ & $722(12.74)$ & 1316(13.96) & 20.7044 & 0.000 \\
\hline
\end{tabular}

BMI: body mass index. 


\begin{tabular}{|c|c|c|c|c|c|}
\hline \multirow[t]{2}{*}{ Variables } & Without dyslipidemia & Dyslipidemia & Total & $x^{2 / t}$ & $P$ \\
\hline & $N=3759$ & $N=5668$ & & & \\
\hline Fetal exposed & 432(11.49) & $665(11.73)$ & 1097(11.64) & & \\
\hline Childhood-exposed & 1431(38.07) & 2332(41.14) & 3763(39.92) & & \\
\hline Adolescence/adult-exposed & $1302(34.64)$ & 1949(34.39) & $3251(34.49)$ & & \\
\hline \multicolumn{6}{|l|}{ BMI } \\
\hline Normal & 2644(70.34) & $2907(51.29)$ & $5551(58.88)$ & 352.436 & 0.000 \\
\hline Overweight & $848(22.56)$ & 1923(33.93) & 2771(29.39) & & \\
\hline Obesity & $267(7.1)$ & $838(14.78)$ & 1105(11.72) & & \\
\hline \multicolumn{6}{|l|}{ Central obesity } \\
\hline Normal & 2632(70.02) & $2840(50.11)$ & $5472(58.05)$ & 367.999 & 0.000 \\
\hline Obesity & $1127(29.98)$ & 2828(49.89) & 3955(41.95) & & \\
\hline
\end{tabular}

Table 3 shows the separate associations of famine exposure, BMI and central obesity with the prevalence of dyslipidemia. Firstly, after controlling for confounding factors including age, educational levels, marital status, place of residence, smoking habits, drinking habits, eating meals, social and leisure activities, the experience of a traumatic event, taking physical activity or exercise, and famine exposure in a multivariable logistic regression model three, higher odds of prevalence of dyslipidemia in the total population were observed with increasing levels of BMI [overweight vs normal:2.06 (95\% Cl 1.86, 2.27); obesity vs normal: $2.82(95 \% \mathrm{Cl} 2.42,3.27)$ ] and WC [overweight vs normal: $2.24(95 \% \mathrm{Cl} 2.05,2.45)$ ] independently of famine stages only (BMI, $P$ for trend = 0.000). When stratified by sex, the results of model three in both males and females were mostly similar to those in the total population. Secondly, after controlling for confounding factors including age, educational levels, marital status, place of residence, smoking habits, drinking habits, eating meals, social and leisure activities, the experience of a traumatic event, taking physical activity or exercise, and BMI in a multivariable logistic regression model three, higher odds of prevalence of dyslipidemia in the total population were observed with famine exposed stages [fatal exposed group vs non-exposed group: 1.32 (95\% $\mathrm{Cl} 1.12,1.56)$; childhood-exposed group vs non-exposed group: 1.49 (95\% Cl 1.30, 1.70); adolescence/adult exposed group vs non-exposed group: 1.49 (95\% $\mathrm{Cl}$ $1.30,1.71)$ ] independently of BMI only $(P$ for trend $=0.000)$. When stratified by sex, the results of model three in females were mostly similar to those in the total population. However, after controlling for confounding factors including age, educational levels, marital status, place of residence, smoking habits, drinking habits, eating meals, social and leisure activities, the experience of a traumatic event, taking physical activity or exercise, and BMI in a multivariable logistic regression model three, associations between famine exposure and dyslipidemia were not observed [fatal exposed group vs non-exposed group: 0.98 (95\% Cl 0.75, 1.28); childhood-exposed group vs non-exposed group: 0.96 (95\% Cl 0.78, 1.19); adolescence/adult exposed group vs non-exposed group: 0.86 $(95 \% \mathrm{Cl} 0.69,1.07)]$ independently of BMI only $(P$ for trend $=0.110)$. Lastly, after controlling for confounding factors including age, educational levels, marital status, place of residence, smoking habits, drinking habits, eating meals, social and leisure activities, the experience of a traumatic event, taking physical activity or exercise, and WC in a multivariable logistic regression model three, higher odds of prevalence of dyslipidemia in the total population were observed with famine exposed stages [fatal exposed group vs non-exposed group: 1.25 (95\% Cl 1.06, 1.47); childhood-exposed group vs non-exposed group: 1.52 (95\% $\mathrm{Cl} 1.34,1.73)$; adolescence/adult exposed group vs non-exposed group: 2.66 ( $95 \% \mathrm{Cl} 2.33,3.03), P$ for trend $=0.000]$ independently of WC only $(P$ for trend $=$ 0.000 ). When stratified by sex, the results of model three in females were mostly similar to those in the total population. Furthermore, after controlling for confounding factors including age, educational levels, marital status, place of residence, smoking habits, drinking habits, eating meals, social and leisure activities, the experience of a traumatic event, taking physical activity or exercise, and WC in a multivariable logistic regression model three, associations between famine exposure and dyslipidemia in male were partly observed [fatal exposed group vs non-exposed group: 0.97 (95\% $\mathrm{Cl} 0.74,1.26$ ); childhoodexposed group vs non-exposed group: 0.91 (95\% Cl 0.74, 1.13); adolescence/adult exposed group vs non-exposed group: 0.73 (95\% $\mathrm{Cl} 0.59,0.91$ )] independently of BMI only ( $P$ for trend $=0.001)$. 
Table 3

Separate associations of famine exposure, $\mathrm{BMI}$, and central obesity with prevalence of dyslipidemia $(\mathrm{N}=9427)$

\begin{tabular}{|c|c|c|c|c|c|c|c|c|}
\hline \multirow{2}{*}{$\begin{array}{l}\text { Variables } \\
\text { Famine exposure }\end{array}$} & \multicolumn{3}{|c|}{ Male (OR and $95 \% \mathrm{Cl}$ for dyslipidemia) } & \multicolumn{3}{|c|}{ Female (OR and $95 \% \mathrm{Cl}$ for dyslipidemia) } & \multicolumn{2}{|c|}{ Total (OR and $95 \% \mathrm{Cl}$} \\
\hline & Model one ${ }^{a}$ & Model two ${ }^{b}$ & Model three $^{c}$ & Model one ${ }^{a}$ & Model two ${ }^{b}$ & Model three $^{c}$ & Model one ${ }^{a}$ & Mo \\
\hline No-exposed & 1.00 (reference) & 1.00 (reference) & 1.00 (reference) & 1.00 (reference) & 1.00 (reference) & 1.00 (reference) & 1.00 (reference) & 1.0 \\
\hline Fetal exposed & $0.92(0.71,1.19)$ & $0.97(0.74,1.27)$ & $0.98(0.75,1.28)$ & $1.54(1.25,1.90)$ & $1.56(1.26,1.93)$ & $1.55(1.25,1.92)$ & $1.27(1.08,1.49)$ & 1.3 \\
\hline Childhood-exposed & $0.84(0.69,1.03)$ & $0.94(0.77,1.17)$ & $0.96(0.78,1.19)$ & $1.86(1.58,2.20)$ & $2.00(1.69,2.38)$ & $1.99(1.68,2.37)$ & $1.34(1.18,1.52)$ & 1.4 \\
\hline $\begin{array}{l}\text { Adolescence/adult- } \\
\text { exposed }\end{array}$ & $0.67(0.55,0.82)$ & $0.85(0.68,1.05)$ & $0.86(0.69,1.07)$ & $2.07(1.74,2.46)$ & $2.43(2.01,2.94)$ & $2.41(1.99,2.93)$ & $1.23(1.08,1.40)$ & 1.4 \\
\hline$P$ for trend & 0.000 & 0.074 & 0.110 & 0.000 & 0.000 & 0.000 & 0.008 & 0.0 \\
\hline Famine exposure & & Model two ${ }^{d}$ & Model three $^{\mathrm{e}}$ & & Model two ${ }^{d}$ & Model three ${ }^{e}$ & & Mo \\
\hline No-exposed & & 1.00 (reference) & 1.00 (reference) & & 1.00 (reference) & 1.00 (reference) & & 1.0 \\
\hline Fetal exposed & & $0.95(0.73,1.25)$ & $0.97(0.74,1.26)$ & & $1.52(1.23,1.88)$ & $1.51(1.22,1.87)$ & & 1.2 \\
\hline Childhood-exposed & & $0.89(0.72,1.10)$ & $0.91(0.74,1.13)$ & & $1.86(1.56,2.20)$ & $1.85(1.56,2.19)$ & & 1.3 \\
\hline $\begin{array}{l}\text { Adolescence/adult- } \\
\text { exposed }\end{array}$ & & $0.72(0.58,0.89)$ & $0.73(0.59,0.91)$ & & $2.04(1.69,2.47)$ & $2.04(1.69,2.47)$ & & 1.2 \\
\hline$P$ for trend & & 0.000 & 0.001 & & 0.000 & 0.000 & & 0.0 \\
\hline BMI & Model one ${ }^{a}$ & Model two ${ }^{f}$ & Model three ${ }^{g}$ & Model one ${ }^{a}$ & Model two ${ }^{f}$ & Model three ${ }^{g}$ & Model one ${ }^{a}$ & Mo \\
\hline $\begin{array}{l}\text { Underweight and } \\
\text { normal }\end{array}$ & 1.00 (reference) & 1.00 (reference) & 1.00 (reference) & 1.00 (reference) & 1.00 (reference) & 1.00 (reference) & 1.00 (reference) & 1.0 \\
\hline Overweight & $2.51(2.17,2.91)$ & $2.42(2.08,2.81)$ & $2.40(2.06,2.79)$ & $1.69(1.49,1.92)$ & $1.79(1.57,2.04)$ & $1.78(1.56,2.03)$ & $2.06(1.87,2.27)$ & 2.1 \\
\hline Obesity & $4.38(3.35,5.72)$ & $4.13(3.16,5.41)$ & $4.10(3.13,5.38)$ & $2.13(1.78,2.55)$ & $2.29(1.91,2.76)$ & $2.26(1.88,2.72)$ & $2.85(2.46,3.31)$ & 2.9 \\
\hline$P$ for trend & 0.000 & 0.000 & 0.000 & 0.000 & 0.000 & 0.000 & 0.000 & 0.0 \\
\hline Central obesity & Model one ${ }^{a}$ & Model two ${ }^{f}$ & Model three $\mathrm{g}$ & Model one ${ }^{a}$ & Model two ${ }^{f}$ & Model three ${ }^{g}$ & Model one ${ }^{a}$ & Mo \\
\hline Normal & 1.00 (reference) & 1.00 (reference) & 1.00 (reference) & 1.00(reference) & 1.00 (reference) & 1.00 (reference) & 1.00 (reference) & 1.0 \\
\hline Obesity & $2.89(2.51,3.33)$ & $2.76(2.39,3.19)$ & $2.73(2.36,3.15)$ & $1.94(1.72,2.18)$ & $1.89(1.68,2.12)$ & $1.88(1.67,2.11)$ & $2.33(2.13,2.54)$ & $2.2^{\prime}$ \\
\hline
\end{tabular}

BMI: body mass index; WC: waist circle; OR: odds ratios; Cl: confidence interval.

Table 4 shows the combined associations of obesity parameters and famine exposure with the prevalence of dyslipidemia in males, females, and the total adults. Compared with the combination of normal BMI/WC level and no-exposed famine stage, all groups trended towards higher or lower odds of prevalence of dyslipidemia. First, in multivariable model three, the greatest increase in odds was observed for the no-exposed stage and obesity combination in males (noexposed stage and obesity in BMl: OR 9.41; $95 \% \mathrm{Cl} 3.65,24.37$; no-exposed stage and obesity in WC: OR 2.89; 95\% Cl 1.91, 4.37). Second, in multivariable model three, the greatest increase in odds was observed for the adolescence/adult exposed stage and obesity combination in females (adolescence/adult exposed stage and obesity in BMI: OR 5.81; 95\% Cl 3.79, 8.90; adolescence/adult exposed stage and obesity in WC: OR 3.70; 95\%Cl 2.87, 4.76). Third, in multivariable-adjusted model three, the highest odds of prevalence of dyslipidemia were observed for the adolescence/adult exposed stage and obesity combination in total adults (childhood-exposed stage and obesity in BMI: OR4.18; $95 \% \mathrm{Cl} 3.18,5.50$; adolescence/adult exposed stage and obesity in WC: OR $2.86 ; 95 \% \mathrm{Cl} 2.38,3.43)$. Finally, combined associations of obesity parameters and famine exposure with the prevalence of dyslipidemia were observed in both males and females $(P$-interaction $=0.000)$. 
Table 4

Combined associations of obesity parameters and famine exposure with prevalence of dyslipidemi

Famine exposure prevalence of dyslipidemia Odds ratio(95\% Cl)

Model one ${ }^{\text {a }}$

BMI levels

\begin{tabular}{|c|c|c|c|c|c|c|c|c|c|}
\hline Male & Normal & overweight & obesity & $\begin{array}{l}P \text { for } \\
\text { trend }\end{array}$ & Normal & overweight & obesity & $\begin{array}{l}P \text { for } \\
\text { trend }\end{array}$ & Nor \\
\hline No-exposed & 1.00 (reference) & $1.85(1.24,2.76)$ & $9.8(3.81,25.23)$ & 0.000 & 1.00 (reference) & $1.82(1.22,2.72)$ & $9.5(3.69,24.46)$ & 0.000 & 1.00 \\
\hline Fetal exposed & $0.95(0.68,1.33)$ & $2.00(1.29,3.10)$ & $4.36(2.05,9.27)$ & 0.000 & $0.95(0.68,1.33)$ & $1.99(1.29,3.09)$ & $4.23(1.99,9.01)$ & 0.000 & 0.96 \\
\hline Childhood-exposed & $0.89(0.68,1.15)$ & $2.08(1.53,2.83)$ & $4.29(2.66,6.91)$ & 0.000 & $0.88(0.68,1.15)$ & $2.03(1.5,2.77)$ & $4.14(2.57,6.67)$ & 0.000 & 0.9 \\
\hline $\begin{array}{l}\text { Adolescence/adult- } \\
\text { exposed }\end{array}$ & $0.74(0.57,0.97)$ & $2.33(1.67,3.24)$ & $1.95(1.20,3.18)$ & 0.000 & $0.74(0.57,0.96)$ & $2.25(1.62,3.14)$ & $1.88(1.16,3.06)$ & 0.000 & 0.75 \\
\hline$P$ for trend & 0.000 & 0.000 & 0.000 & & 0.000 & 0.000 & 0.000 & & 0.00 \\
\hline \multirow[t]{3}{*}{$P$-interaction } & & 0.000 & & & & 0.000 & & & \\
\hline & WC levels & & & & WC levels & & & & WC \\
\hline & Normal & Central obesity & & & Normal & Central obesity & & & Nor \\
\hline No-exposed & 1.00 (reference) & $3.00(1.99,4.53)$ & & & 1.00 (reference) & $2.93(1.94,4.42)$ & & & 1.0( \\
\hline Fetal exposed & $0.97(0.71,1.33)$ & $2.72(1.76,4.20)$ & & & $0.97(0.71,1.33)$ & $2.66(1.72,4.12)$ & & & $0.9 \varepsilon$ \\
\hline Childhood-exposed & $0.91(0.71,1.16)$ & $2.55(1.90,3.42)$ & & & $0.91(0.71,1.16)$ & $2.46(1.84,3.31)$ & & & $0.9:$ \\
\hline $\begin{array}{l}\text { Adolescence/adult- } \\
\text { exposed }\end{array}$ & $0.73(0.57,0.94)$ & $2.10(1.56,2.83)$ & & & $0.73(0.57,0.94)$ & $2.04(1.51,2.75)$ & & & $0.7<$ \\
\hline$P$ for trend & 0.000 & 0.000 & & & 0.000 & 0.000 & & & 0.00 \\
\hline$P$-interaction & 0.000 & & & & 0.000 & & & & $0.0 c$ \\
\hline Female & Normal & overweight & obesity & $\begin{array}{l}P \text { for } \\
\text { trend }\end{array}$ & Normal & overweight & obesity & $\begin{array}{l}P \text { for } \\
\text { trend }\end{array}$ & Nor \\
\hline No-exposed & 1.00 (reference) & $1.95(1.43,2.66)$ & $1.68(1.14,2.47)$ & 0.000 & 1.00 (reference) & $1.95(1.43,2.65)$ & $1.67(1.14,2.46)$ & 0.000 & 1.00 \\
\hline Fetal exposed & $1.56(1.14,2.12)$ & $2.45(1.76,3.41)$ & $4.04(2.49,6.54)$ & 0.000 & $1.56(1.14,2.13)$ & $2.45(1.76,3.41)$ & $4.03(2.48,6.52)$ & 0.000 & 1.5 \\
\hline Childhood-exposed & $1.95(1.54,2.48)$ & $3.29(2.52,4.29)$ & $4.73(3.37,6.64)$ & 0.000 & $1.96(1.54,2.50)$ & $3.29(2.52,4.29)$ & $4.71(3.35,6.62)$ & 0.000 & $1.9 €$ \\
\hline $\begin{array}{l}\text { Adolescence/adult- } \\
\text { exposed }\end{array}$ & $2.18(1.71,2.78)$ & $4.29(3.19,5.77)$ & $5.91(3.88,9.01)$ & 0.000 & $2.21(1.71,2.84)$ & $4.30(3.18,5.82)$ & $5.91(3.86,9.05)$ & 0.000 & 2.20 \\
\hline$P$ for trend & 0.000 & 0.000 & 0.000 & & 0.000 & 0.000 & 0.000 & & 0.00 \\
\hline$P$-interaction & & 0.000 & & & & 0.000 & & & \\
\hline
\end{tabular}

WC levels

Normal Central obesity

No-exposed $\quad 1.00$ (reference) $1.63(1.23,2.15)$

Fetal exposed $\quad 1.42(1.06,1.91) \quad 2.65(1.96,3.58)$

Childhood-exposed $\quad 1.69(1.34,2.12) \quad 3.29(2.60,4.16)$

Adolescence/adult- $\quad 1.78(1.40,2.28) \quad 3.66(2.87,4.67)$

exposed

$P$ for trend

$P$-interaction

Total

rations




\begin{tabular}{|c|c|c|c|c|c|c|c|c|c|}
\hline \multirow[t]{3}{*}{ Famine exposure } & \multicolumn{9}{|c|}{ prevalence of dyslipidemia Odds ratio $(95 \% C l)$} \\
\hline & \multicolumn{4}{|l|}{ Model one ${ }^{a}$} & \multicolumn{4}{|l|}{ Model two ${ }^{b}$} & \multirow{2}{*}{$\frac{\mathrm{Mol}}{\mathrm{BM}}$} \\
\hline & BMI levels & & & & BMI levels & & & & \\
\hline No-exposed & 1.00 (reference) & $1.85(1.45,2.36)$ & $2.19(1.58,3.04)$ & 0.000 & 1.00 (reference) & $1.85(1.45,2.36)$ & $2.17(1.57,3.02)$ & 0.000 & $1.0 c$ \\
\hline Fetal exposed & $1.26(1.00,1.58)$ & $2.20(1.69,2.86)$ & $3.91(2.62,5.84)$ & 0.000 & $1.26(1.01,1.58)$ & $2.21(1.70,2.87)$ & $3.86(2.58,5.77)$ & 0.000 & $1.2^{-}$ \\
\hline Childhood-exposed & $1.35(1.13,1.60)$ & $2.71(2.22,3.30)$ & $4.37(3.33,5.75)$ & 0.000 & $1.33(1.11,1.58)$ & $2.63(2.15,3.21)$ & $4.23(3.22,5.57)$ & 0.000 & 1.36 \\
\hline $\begin{array}{l}\text { Adolescence/adult- } \\
\text { exposed }\end{array}$ & $1.26(1.06,1.50)$ & $3.29(2.64,4.10)$ & $3.78(2.76,5.18)$ & 0.000 & $1.21(1.02,1.45)$ & $3.15(2.52,3.93)$ & $3.55(2.58,4.88)$ & 0.000 & $1.2 ?$ \\
\hline$P$ for trend & 0.000 & 0.000 & 0.000 & & 0.000 & 0.000 & 0.000 & & $0.0 c$ \\
\hline \multirow[t]{3}{*}{$P$-interaction } & & 0.000 & & & & 0.000 & & & \\
\hline & \multicolumn{3}{|l|}{ WC levels } & $\begin{array}{l}\text { WC } \\
\text { levels }\end{array}$ & & & \multicolumn{3}{|l|}{ WC levels } \\
\hline & Normal & Central obesity & & & Normal & Central obesity & & & Nor \\
\hline No-exposed & 1.00(reference) & $1.85(1.48,2.31)$ & & & 1.00(reference) & $1.84(1.47,2.30)$ & & & $1.0 c$ \\
\hline Fetal exposed & $1.21(0.97,1.49)$ & $2.54(1.99,3.24)$ & & & $1.21(0.98,1.50)$ & $2.53(1.98,3.23)$ & & & $1.2 i$ \\
\hline Childhood-exposed & $1.25(1.06,1.47)$ & $2.92(2.43,3.50)$ & & & $1.25(1.06,1.47)$ & $2.85(2.38,3.43)$ & & & $1.2 \varepsilon$ \\
\hline $\begin{array}{l}\text { Adolescence/adult- } \\
\text { exposed }\end{array}$ & $1.09(0.92,1.29)$ & $2.93(2.43,3.53)$ & & & $1.08(0.91,1.29)$ & $2.84(2.34,3.43)$ & & & 1.11 \\
\hline$P$ for trend & 0.000 & 0.000 & & & 0.000 & 0.000 & & & $0.0 c$ \\
\hline$P$-interaction & 0.000 & & & & 0.000 & & & & $0.0 c$ \\
\hline
\end{tabular}

BMI: body mass index; WC: waist circle.

a Unadjusted; age-adjusted by design

${ }^{b}$ Adjusted for age, educational levels, marital status, place of residence;

${ }^{c}$ Adjusted for age, educational levels, marital status, place of residence, smoking habits, drinking habits, eating meals, social and leisure activities, experienct

\section{Discussion}

In our study, we found that the participants exposed to famine in early life had increased risk of dyslipidemia in females, but decreased risk of dyslipidemia in males. After adjustment for observed confounders, including age, educational levels, marital status, place of residence, smoking habits, drinking habits, eating meals, social and leisure activities, the experience of a traumatic event, taking physical activity or exercise, and obesity parameters, the associations still existed both in males and females. Additionally, the study showed that there were increased trends in the associations of BMI/WC with dyslipidemia. After adjustment for observed confounders, including age, educational levels, marital status, place of residence, smoking habits, drinking habits, eating meals, social and leisure activities, the experience of a traumatic event, taking physical activity or exercise, and famine exposure, the associations still existed both in males and females. In general, combined associations of obesity parameters and famine exposure with the prevalence of dyslipidemia were observed in our study. When stratified by sex, males and females differed in most of the risks of dyslipidemia from the opposite sex.

The Chinese famine lasted from the late1950s to the early 1960s, caused over 30 million excess deaths in most areas[31]. Together with present study, most studies have assessed the associations of famine exposure during early life with risk of dyslipidemia in adults, and no consistent associations were observed. Therefore, this research attempted to examine the association analysis between obesity parameters and dyslipidemia based on a population-based crosssectional study from CHARLS. Our data support a strongly positive combined effect of famine exposure and obesity parameters on dyslipidemia in middleaged and elderly Chinese. Both nutrition intervention for exposure to the famine in early life and weight reduction in later life may be required to substantially reduce the risk of dyslipidemia in later life.

As the worst famine, the survivors might be healthier than the frail members were kicked out, a finding that is in line with Darwin' s theory of survival of the fittest [32]. In this case, the participants exposed to famine in early life should decrease the risk of dyslipidemia in adults. Sex difference in our study is to be expected. Exposure to famine in adolescence/adults decreased the risk of dyslipidemia in males. However, exposure to famine in early life increased the risk of dyslipidemia in females. When facing the later "rich" environment, the risk of dyslipidemia may be increased. The sex-specific effect might also be associated with the hypothesis that parents in China prefer boys to girls traditionally or survivors' bias.

The outcomes are partly in line with previous studies. X. Xin, W. Wang, H. Xu, et al [18] found that exposure to the Chinese famine in early life was associated with an increased risk of dyslipidemia in adulthood. However, another study[17] did not find the association between exposure to famine early in life and the increased risk of dyslipidemia in adults. However, when stratified by sex, a significantly increased risk of dyslipidemia in females in the fetal-exposure group compared to females in the nonexposed group was found, but the significant difference was not present among males. Z. Wang, C. Li, Z. Yang, et al 
[19]reported that exposure to severe Chinese famine early in life was associated with the higher dyslipidemia risk in females in adult, but not in males in adult. Such discrepancies between those studies may be a result of methodological differences in definitions of famine exposure groups and the different sample selection effect. Additionally, these studies have been criticized for not being adjusted the effect of age. To control the potential age confounding, we categorized the famine exposure into four exposure cohorts [no-exposed stage (born between1963 and 1974), fetal exposed stage (born between1959 and 1962), childhood exposed stage (born between 1949 and 1958), adolescence/adult exposed stage (born between1921 and 1948 )] based on the birth year. Our results suggested that early famine exposure was associated with an increased risk of dyslipidemia in females. However, exposure to famine in adolescence/adults decreased the risk of dyslipidemia in males. The sex difference of early life famine exposure and dyslipidemia were common in previous studies $[17,19]$. Furthermore, exposure to famine during early life exerted more deleterious effects on women than men. This could be explained by the fact the women may suffer more than men during the famine because of the dominance of a patriarchal mentality in China[33]. The potential mechanisms of the relationship between famine exposure in early life and the increased risk of dyslipidemia in later life were still not fully understood. Exposure to famine in early life may lead to altered lipid profiles in adulthood, and exposure to famine during the fetal period significantly increased the risk. "Fetal Origins hypothesis" was that early adaptations in response to malnutrition resulted in metabolic changes, which were beneficial for short-term survival, but could increase the risk of chronic diseases in adults [34,35]. Animal model[36] has proved that malnutrition in early life could result in modified cholesterol synthesis and elevated plasma cholesterol concentrations. In addition, epigenetic might play a role in the association between famine exposure in early life and dyslipidemia in adults [37].

In our research, participants who were overweight/obesity, and exposed to famine in early life tended to have a higher risk of dyslipidemia prevalence. The results indicated the good nutrition in adults did not match poor nutrition in early life, which might elevate the relative risk of early life in later life. Furthermore, our data support a strongly positive combined effect of famine exposure and obesity parameters on dyslipidemia in middle-aged and elderly Chinese. However, the previous studies had only focused on the relationship between famine exposure and health outcomes in late adolescence and adulthood. Most of the previous studies meant exactly that: famine exposure was at a higher risk for health outcomes in late adolescence and adulthood. Exposure to Chinese famine in early life was related to increased risk of metabolic syndrome[30, 38-43], weight gain [44-49], diabetes[10, 50-64], hypertension[13, 14, 65-74], cognitive decline[15, 16, 75-79], depressive syndrome[31, 75, 80, 81].Interestingly, we found that males who were normal weight and exposed to famine in adolescence/adult tended to have a lower risk of dyslipidemia prevalence, and males who were obesity, and no-exposed to famine tended to have the greatest risk of dyslipidemia prevalence. The combined effects differed from the trend of risk in females.

There were several limitations to the study. First, selection bias was to be considered: famine may weed out the frail members of the population and leave the healthier ones. Second, famine exposure for each individual was unknown. Third, not all families were equally affected by famine exposure. However, the results provided large cohort data that could be explored further in the combined effect of famine exposure and obesity parameters on dyslipidemia. Moreover, a significant strength of the study is the large sample of 9427 middle-aged and older Chinese. Another strength is the analytical method the controlled of the potential confounders.

\section{Conclusions}

Our data support individual and combined associations of obesity parameters and famine exposure with the prevalence of dyslipidemia in middle-aged and elderly Chinese. Both nutrition intervention for exposure to the famine in early life and weight reduction in later life may be required to substantially reduce the risk of dyslipidemia in later life.

\section{Abbreviations}

World Health Organization, WHO; CHARLS, China Health and Retirement Longitudinal Study; BMI, body mass index; waist circle, WC; BP, blood pressure; DBP, diastolic blood pressure; SBP, systolic blood pressure; M, mean; unstandardized, B; CDC, Centers for Disease Control and Prevention; NSFC, The National Natural Science Foundation of China; National Institute on Aging, NIA; World Bank, WB; Frozen plasma low-density lipoprotein cholesterol, LDL-C; high-density lipoprotein cholesterol, HDL-c; triglycerides, TG; total cholesterol, TC; National Cholesterol Education Programme, NCEP; Adult Treatment Panel III, NCEP ATP III; CVD, cardiovascular disease.

\section{Declarations}

\section{Ethics approval and consent to participate}

All data are openly published as microdata at http://opendata.pku.edu.cn/dataverse/CHARLS with no direct contact with all participants. The Ethics Committee of the China Centre for Economic Research at Peking University approved this study. All the research program for involving humans was in accordance with guidelines of national/international/institutional or Declaration of Helsinki. Written informed consent was obtained from all participants.

\section{Consent to publish}

Not applicable.

\section{Availability of data and material}

The datasets generated and/or analysed during the current study are available in the CHARLS repository, http://opendata.pku.edu.cn/dataverse/CHARLS.

\section{Competing interests}


All authors report no potential conflict of interest relevant to the research.

Funding

This work was supported by the NSFC (70910107022, 71130002) and National Institute on Aging (R03-TW008358-01; R01-AG037031-03S1), World Bank (7159234), and the publication fee was supported by the Support Program for Outstanding Young Talents from the Universities and Colleges of Anhui Province for Lin Zhang(gxyqZD2021118), and the Key Research Base of Humanities and Social Sciences of Universities of Anhui Province (SK2019A0223) for Wang Congzhi.

\section{Authors' contributions}

Conceived and designed the research: LZ and H-y L. Wrote the paper: LZ. Analyzed the data: LZ. Revised the paper: LZ, J-I L, L-I G, L Y, C-z W, TY, Dm Z, H-h W,

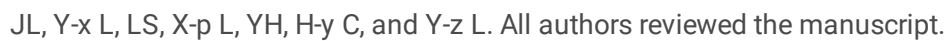

\section{Acknowledgements}

We thank the members of the research as well as all participants for their contribution.

\section{Author affiliations}

${ }^{1}$ Department of Internal Medicine Nursing, School of Nursing, Wannan Medical College, 22 Wenchang West Road, Higher Education Park, Wuhu City, An Hui Province, P.R.China. ${ }^{2}$ Obstetrics and Gynecology Nursing, School of Nursing, Wannan Medical College, 22 Wenchang West Road, Higher Education Park, Wuhu City, An Hui Province, P.R.China. ${ }^{3}$ Department of Pediatric Nursing, School of Nursing, Wannan Medical College, 22 Wenchang West Road, Higher Education Park, Wuhu City, An Hui Province, P.R.China. ${ }^{4}$ Department of Surgical Nursing, School of Nursing, Wannan Medical College, 22 Wenchang West Road, Higher Education Park, Wuhu City, An Hui Province, P.R.China. ${ }^{5}$ Department of Emergency and Critical Care Nursing, School of Nursing, Wannan Medical College, 22 Wenchang West Road, Higher Education Park, Wuhu City, An Hui Province, P.R.China. ${ }^{6}$ Rehabilitation Nursing, School of Nursing, Wanna Medical college, 22 Wenchang West Road, Higher Education Park, Wuhu City, An Hui Province, P.R.China. ${ }^{7}$ Department of nursing, Yijishan Hospital, the First Affiliated Hospital of Wannan Medical College, Zheshan West Road, Yijishan District, Wuhu City, Anhui Province. ${ }^{8}$ Department of nursing, Health Center of Wannan Medical College, Zheshan West Road, Yijishan District, Wuhu City, Anhui Province

\section{References}

1. Mendis S, Davis S, Norrving B: Organizational update: the world health organization global status report on noncommunicable diseases 2014; one more landmark step in the combat against stroke and vascular disease. Stroke 2015, 46(5):e121-122.

2. Pan L, Yang Z, Wu Y, Yin RX, Liao Y, Wang J, Gao B, Zhang L: The prevalence, awareness, treatment and control of dyslipidemia among adults in China. Atherosclerosis 2016, 248:2-9.

3. Opoku S, Gan Y, Fu W, Chen D, Addo-Yobo E, Trofimovitch D, Yue W, Yan F, Wang Z, Lu Z: Prevalence and risk factors for dyslipidemia among adults in rural and urban China: findings from the China National Stroke Screening and prevention project (CNSSPP). BMC Public Health 2019, 19(1):1500.

4. Hernández-Reyes A, Vidal Á, Moreno-Ortega A: Waist Circumference as a Preventive Tool of Atherogenic Dyslipidemia and Obesity-Associated Cardiovascular Risk in Young Adults Males: A Cross-Sectional Pilot Study. 2020, 10(12).

5. Vekic J, Zeljkovic A, Stefanovic A, Jelic-Ivanovic Z, Spasojevic-Kalimanovska V: Obesity and dyslipidemia. Metabolism: clinical and experimental 2019, 92:71-81.

6. Moreno-Rojas R, Khan SH, Shahid R, Fazal N, ljaz A: Comparison of Various Abdominal Obesity Measures for Predicting Metabolic Syndrome, Diabetes, Nephropathy, and Dyslipidemia. Diagnostics (Basel, Switzerland) 2019, 29(12):1159-1164.

7. Zhou LY, Deng MQ, Zhang Q, Xiao XH: Early-life nutrition and metabolic disorders in later life: a new perspective on energy metabolism. Chinese medical journal 2020, 133(16):1961-1970.

8. Qin LL, Luo BA, Gao F: Effect of Exposure to Famine during Early Life on Risk of Metabolic Syndrome in Adulthood: A Meta-Analysis. 2020, 2020:3251275.

9. Feng XL, Liu JH, Wang N, Wang X, Li Q, Han B, Chen Y, Zhu C, Chen Y, Lin D et al: The famine exposure in early life and metabolic syndrome in adulthood. Journal of diabetes research 2017, 36(1):253-259.

10. Ma RCW, Zimmet P, Liu H, Chen X, Shi T, Qu G, Zhao T, Xuan K, Sun Y: Association of famine exposure with the risk of type 2 diabetes: A meta-analysis. Journal of diabetes 2020, 39(6):1717-1723.

11. Arage G, Belachew T, Hassen H, Abera M, Abdulhay F, Abdulahi M, Hassen Abate K: Effects of prenatal exposure to the 1983-1985 Ethiopian great famine on the metabolic syndrome in adults: a historical cohort study. The British journal of nutrition 2020, 124(10):1052-1060.

12. Pang Z, Qiao Q, Diabetes Prevention Program O, Wang Z: Chinese famine exposure in infancy and metabolic syndrome in adulthood: results from the China health and retirement longitudinal study. Journal of diabetes research 2019, 73(5):724-732.

13. Zhao R, Duan X, Wu Y, Zhang Q, Chen Y: Association of exposure to Chinese famine in early life with the incidence of hypertension in adulthood: A 22-year cohort study. Nutrition, metabolism, and cardiovascular diseases: NMCD 2019, 29(11):1237-1244.

14. Xin X, Yao J, Yang F, Zhang D: Famine exposure during early life and risk of hypertension in adulthood: A meta-analysis. Critical reviews in food science and nutrition 2018, 58(14):2306-2313. 
15. Arage G, Belachew T, Abera M, Abdulhay F, Abdulahi M, Hassen Abate K: Consequences of early life exposure to the 1983-1985 Ethiopian Great Famine on cognitive function in adults: a historical cohort study. BMJ open 2020, 10(9):e038977.

16. Rong H, Lai X, Mahmoudi E, Fang H: Early-Life Exposure to the Chinese Famine and Risk of Cognitive Decline. Journal of clinical medicine 2019, 8(4).

17. Yao H, Li L: Famine Exposure during the Fetal Period Increased the Risk of Dyslipidemia in Female Adults. Lipids 2019, 54(5):301-309.

18. Xin X, Wang W, Xu H, Li Z, Zhang D: Exposure to Chinese famine in early life and the risk of dyslipidemia in adulthood. 2019, 58(1):391-398.

19. Wang Z, Li C, Yang Z, Ma J, Zou Z: Fetal and infant exposure to severe Chinese famine increases the risk of adult dyslipidemia: Results from the China health and retirement longitudinal study. European journal of nutrition 2017, 17(1):488.

20. Li C, Liu T, Sun W, Wu L, Zou ZY: Prevalence and risk factors of arthritis in a middle-aged and older Chinese population: the China health and retirement longitudinal study. Rheumatology 2015, 54(4):697-706.

21. Zhang L, Liu K, Li H, Li D, Chen Z, Zhang LL, Guo LL: Relationship between body mass index and depressive symptoms: the "fat and jolly" hypothesis for the middle-aged and elderly in China. BMC public health 2016, 16(1):1201.

22. Zhang L, Li JL, Zhang LL, Guo LL, Li H, Li D: No association between C-reactive protein and depressive symptoms among the middle-aged and elderly in China Evidence from the China Health and Retirement Longitudinal Study. Medicine 2018, 97(38).

23. Zhang L, Li JL, Zhang LL, Guo LL, Li H, Li D: Association and Interaction Analysis of Body Mass Index and Triglycerides Level with Blood Pressure in Elderly Individuals in China. BioMed research international 2018, 2018:8934534.

24. Zhang L, Li JL, Zhang LL, Guo LL, Li H, Yan WZ, Li D: Relationship between adiposity parameters and cognition: the "fat and jolly" hypothesis in middleaged and elderly people in China. Medicine 2019, 98(10).

25. Zhang L, Li JL, Zhang LL, Guo LL, Li H, Li D: Body mass index and serum uric acid level: Individual and combined effects on blood pressure in middleaged and older individuals in China. Medicine (Baltimore) 2020, 99(9):e19418.

26. Zhang L, Li JL, Guo LL, Li H, Li D, Xu G: The interaction between serum uric acid and triglycerides level on blood pressure in middle-aged and elderly individuals in China: result from a large national cohort study. BMC cardiovascular disorders 2020, 20(1):174.

27. Zhou BF: Effect of body mass index on all-cause mortality and incidence of cardiovascular diseases-report for meta-analysis of prospective studies open optimal cut-off points of body mass index in Chinese adults. Biomedical and environmental sciences: BES 2002, 15(3):245-252.

28. Zhou HC, Lai YX, Shan ZY, Jia WP, Yang WY, Lu JM, Weng JP, Ji LN, Liu J, Tian HM et al: Effectiveness of different waist circumference cut-off values in predicting metabolic syndrome prevalence and risk factors in adults in China. Biomedical and environmental sciences: BES 2014, 27(5):325-334.

29. Wang D, Wan S, Liu P, Meng F, Ren B, Qu M, Wu H, Zhou Z, Jin M, Shen H et al: Associations between water iodine concentration and the prevalence of dyslipidemia in Chinese adults: A cross-sectional study. Ecotoxicology and environmental safety 2021, 208:111682.

30. Wang N, Wang X, Li Q, Han B, Chen Y, Zhu C, Chen Y, Lin D, Wang B, Jensen MD et al: The famine exposure in early life and metabolic syndrome in adulthood. Clinical nutrition (Edinburgh, Scotland) 2017, 36(1):253-259.

31. Li Y, Zhao L, Yu D, Ding G: Exposure to the Chinese famine in early life and depression in adulthood. Psychology, health \& medicine 2018, 23(8):952-957.

32. Song S: Does famine have a long-term effect on cohort mortality? Evidence from the 1959-1961 great leap forward famine in China. Journal of biosocial science 2009, 41(4):469-491.

33. Khanal P, Johnsen L, Axel AM, Hansen PW, Kongsted AH, Lyckegaard NB, Nielsen MO: Long-Term Impacts of Foetal Malnutrition Followed by Early Postnatal Obesity on Fat Distribution Pattem and Metabolic Adaptability in Adult Sheep. PloS one 2016, 11(6):e0156700.

34. Gluckman PD, Hanson MA, Cooper C, Thornburg KL: Effect of in utero and early-life conditions on adult health and disease. The New England journal of medicine 2008, 359(1):61-73.

35. Gluckman PD, Hanson MA, Bateson P, Beedle AS, Law CM, Bhutta ZA, Anokhin KV, Bougnères P, Chandak GR, Dasgupta $P$ et al: Towards a new developmental synthesis: adaptive developmental plasticity and human disease. Lancet (London, England) 2009, 373(9675):1654-1657.

36. Lucas A, Baker BA, Desai M, Hales CN: Nutrition in pregnant or lactating rats programs lipid metabolism in the offspring. The British journal of nutrition 1996, 76(4):605-612.

37. Aagaard-Tillery KM, Grove K, Bishop J, Ke X, Fu Q, McKnight R, Lane RH: Developmental origins of disease and determinants of chromatin structure: maternal diet modifies the primate fetal epigenome. Journal of molecular endocrinology 2008, 41(2):91-102.

38. Peng Y, Hai M, Li P, Chen Y: Association of Exposure to Chinese Famine in Early Life with the Risk of Metabolic Syndrome in Adulthood. Annals of nutrition \& metabolism 2020, 76(2):140-146.

39. Feng XL, Liu JH, Yan S, Hou W, Wu H, Jiang W, Li Y, Zhang Y, Li H, Yang S et al: Prenatal exposure to the Chinese famine and the risk of metabolic syndrome in adulthood across consecutive generations. Journal of diabetes research 2020, 74(8):1229-1236.

40. Ning F, Ren J, Song X, Zhang D, Liu L, Zhang L, Sun J, Zhang D: Famine Exposure in Early Life and Risk of Metabolic Syndrome in Adulthood: Comparisons of Different Metabolic Syndrome Definitions. 2019, 2019:7954856.

41. Zheng X, Wang Y, Ren W, Luo R, Zhang S, Zhang JH, Zeng Q: Risk of metabolic syndrome in adults exposed to the great Chinese famine during the fetal life and early childhood. European journal of clinical nutrition 2012, 66(2):231-236.

42. Li Y, Jaddoe VW, Qi L, He Y, Wang D, Lai J, Zhang J, Fu P, Yang X, Hu FB: Exposure to the chinese famine in early life and the risk of metabolic syndrome in adulthood. Diabetes care 2011, 34(4):1014-1018.

43. de Rooij SR, Painter RC, Holleman F, Bossuyt PM, Roseboom TJ: The metabolic syndrome in adults prenatally exposed to the Dutch famine. The American journal of clinical nutrition 2007, 86(4):1219-1224.

Page $14 / 16$ 
44. Liu L, Pang ZC, Sun JP, Xue B, Wang SJ, Ning F, Qiao Q: Exposure to famine in early life and the risk of obesity in adulthood in Qingdao: Evidence from the 1959-1961 Chinese famine. Nutrition, metabolism, and cardiovascular diseases: NMCD 2017, 27(2):154-160.

45. Chang X, Song P, Wang M, An L: The Risks of Overweight, Obesity and Abdominal Obesity in Middle Age after Exposure to Famine in Early Life: Evidence from the China's 1959-1961 Famine. The journal of nutrition, health \& aging 2018, 22(10):1198-1204.

46. Meng R, Lv J, Yu C, Guo Y, Bian Z, Yang L, Chen Y, Zhang H, Chen X, Chen J et al: Prenatal famine exposure, adulthood obesity patterns and risk of type 2 diabetes. International journal of epidemiology 2018, 47(2):399-408.

47. Zhou J, Zhang L, Xuan P, Fan Y, Yang L, Hu C, Bo Q, Wang G, Sheng J, Wang S: The relationship between famine exposure during early life and body mass index in adulthood: A systematic review and meta-analysis. PloS one 2018, 13(2):e0192212.

48. Fang Z, Chen C, Wang H, Tang K: Association Between Fetal Exposure to Famine and Anthropometric Measures in Adulthood: A Regression Discontinuity Approach. Obesity (Silver Spring, Md) 2020, 28(5):962-969.

49. Song C, Wang M, Chen Z, Yao Y, Feng G, Ma Y, Fan J, Liu A: Fetal Exposure to Chinese Famine Increases Obesity Risk in Adulthood. International journal of environmental research and public health 2020, 17(10).

50. Lu J: Early Life Famine Exposure, Ideal Cardiovascular Health Metrics, and Risk of Incident Diabetes: Findings From the 4C Study. The British journal of nutrition 2020, 43(8):1902-1909.

51. Ji L, Shi Z: Early life exposure to 1959-1961 Chinese famine exacerbates association between diabetes and cardiovascular disease. Nature reviews Endocrinology 2020, 12(2):134-141.

52. Hu X, Wen J, Yu W, Yang L, Pan W, Xu K, Chen X, Li Q, Chen G, Gu X: Associations of early-life exposure to famine with abdominal fat accumulation are independent of family history of diabetes and physical activity. 2020:1-8.

53. Li C, Tobi EW, Heijmans BT, Lumey LH: The effect of the Chinese Famine on type 2 diabetes mellitus epidemics. 2019, 15(6):313-314.

54. Li C, Lumey LH: Interaction or mediation by adult obesity of the relation between fetal famine exposure and type 2 diabetes? Nature reviews Endocrinology 2019, 48(2):654-656.

55. Wang Z, Zou Z, Yang Z, Dong Y, Song J, Dong B, Ma J: The association between fetal-stage exposure to the China famine and risk of diabetes mellitus in adulthood: results from the China health and retirement longitudinal study. 2018, 18(1):1205

56. Sun Y, Zhang L, Duan W, Meng X, Jia C: Association between famine exposure in early life and type 2 diabetes mellitus and hyperglycemia in adulthood: Results from the China Health And Retirement Longitudinal Study (CHARLS). Journal of diabetes 2018, 10(9):724-733.

57. Li T, Zhang Y, Wang J, Xu D, Yin Z, Chen H, Lv Y, Luo J, Zeng Y, Liu Y et al: All-cause mortality risk associated with long-term exposure to ambient PM(2.5) in China: a cohort study. The Lancet Public health 2018, 3(10):e470-e477.

58. Wang N, Cheng J, Han B, Li Q, Chen Y, Xia F, Jiang B, Jensen MD, Lu Y: Exposure to severe famine in the prenatal or postnatal period and the development of diabetes in adulthood: an observational study. Diabetologia 2017, 60(2):262-269.

59. Li J, Liu S, Li S, Feng R, Na L, Chu X, Wu X, Niu Y, Sun Z, Han T et al: Prenatal exposure to famine and the development of hyperglycemia and type 2 diabetes in adulthood across consecutive generations: a population-based cohort study of families in Suihua, China. The American journal of clinical nutrition 2017, 105(1):221-227.

60. Wang J, Li Y, Han X, Liu B, Hu H, Wang F, Li X, Yang K, Yuan J, Yao P et al: Exposure to the Chinese Famine in Childhood Increases Type 2 Diabetes Risk in Adults. The Journal of nutrition 2016, 146(11):2289-2295.

61. Lumey LH, Khalangot MD, Vaiserman AM: Association between type 2 diabetes and prenatal exposure to the Ukraine famine of 1932-33: a retrospective cohort study. The lancet Diabetes \& endocrinology 2015, 3(10):787-794.

62. van Abeelen AF, Elias SG, Bossuyt PM, Grobbee DE, van der Schouw YT, Roseboom TJ, Uiterwaal CS: Famine exposure in the young and the risk of type 2 diabetes in adulthood. Diabetes 2012, 61(9):2255-2260

63. Portrait F, Teeuwiszen E, Deeg D: Early life undernutrition and chronic diseases at older ages: the effects of the Dutch famine on cardiovascular diseases and diabetes. Social science \& medicine (1982) 2011, 73(5):711-718.

64. Li Y, He Y, Qi L, Jaddoe VW, Feskens EJ, Yang X, Ma G, Hu FB: Exposure to the Chinese famine in early life and the risk of hyperglycemia and type 2 diabetes in adulthood. Diabetes 2010, 59(10):2400-2406.

65. Li Y, Jaddoe VW, Qi L, He Y, Lai J, Wang J, Zhang J, Hu Y, Ding EL, Yang X et al: Exposure to the Chinese famine in early life and the risk of hypertension in adulthood. Journal of hypertension 2011, 29(6):1085-1092.

66. van Abeelen AFM, de Rooij SR, Osmond C, Painter RC, Veenendaal MVE, Bossuyt PMM, Elias SG, Grobbee DE, van der Schouw YT, Barker DJP et al: The sex-specific effects of famine on the association between placental size and later hypertension. Placenta 2011, 32(9):694-698.

67. Wang PX, Wang JJ, Lei YX, Xiao L, Luo ZC: Impact of fetal and infant exposure to the Chinese Great Famine on the risk of hypertension in adulthood. PloS one 2012, 7(11):e49720.

68. Chen H, Nembhard WN, Stockwell HG: Sex-specific effects of fetal exposure to the 1959-1961 Chinese famine on risk of adult hypertension. Maternal and child health journal 2014, 18(3):527-533.

69. Wang Z, Li C, Yang Z, Zou Z, Ma J: Infant exposure to Chinese famine increased the risk of hypertension in adulthood: results from the China Health and Retirement Longitudinal Study. BMC Public Health 2016, 16:435

70. Liu L, Xu X, Zeng H, Zhang Y, Shi Z, Zhang F, Cao X, Xie YJ, Reis C, Zhao Y: Increase in the prevalence of hypertension among adults exposed to the Great Chinese Famine during early life. Environmental health and preventive medicine 2017, 22(1):64.

71. Wu L, Feng X, He A, Ding Y, Zhou X, Xu Z: Prenatal exposure to the Great Chinese Famine and mid-age hypertension. 2017, 12(5):e0176413. 
72. Yu C, Wang J, Li Y, Han X, Hu H, Wang F, Yuan J, Yao P, Miao X, Wei S et al: Exposure to the Chinese famine in early life and hypertension prevalence risk in adults. PloS one 2017, 35(1):63-68.

73. Liu L, Xu X, Zeng H, Zhang Y, Shi Z, Zhang F, Cao X, Xie YJ, Reis C, Zhao Y: Correction to: Increase in the prevalence of hypertension among adults exposed to the great Chinese famine during early life. Environmental health and preventive medicine 2018, 23(1):11.

74. Shi Z, Nicholls SJ, Taylor AW, Magliano DJ, Appleton S, Zimmet P: Early life exposure to Chinese famine modifies the association between hypertension and cardiovascular disease. Journal of hypertension 2018, 36(1):54-60.

75. Li C, Miles T, Shen L, Shen Y, Liu T, Zhang M, Li S, Huang C: Early-life exposure to severe famine and subsequent risk of depressive symptoms in late adulthood: the China Health and Retirement Longitudinal Study. The British journal of psychiatry: the journal of mental science 2018, 213(4):579-586.

76. He P, Liu L, Salas JMI, Guo C, Cheng Y, Chen G, Zheng X: Prenatal malnutrition and adult cognitive impairment: a natural experiment from the 1959-1961 Chinese famine. The British journal of nutrition 2018, 120(2):198-203.

77. Rong H, Xi Y, An Y, Tao L, Zhang X, Yu H, Wang Y, Qin Z, Xiao R: The Correlation between Early Stages of Life Exposed to Chinese Famine and Cognitive Decline in Adulthood: Nutrition of Adulthood Plays an Important Role in the Link? Frontiers in aging neuroscience 2017, 9:444.

78. Wang C, An Y, Yu H, Feng L, Liu Q, Lu Y, Wang H, Xiao R: Association between Exposure to the Chinese Famine in Different Stages of Early Life and Decline in Cognitive Functioning in Adulthood. Frontiers in behavioral neuroscience 2016, 10:146.

79. Li J, Na L, Ma H, Zhang Z, Li T, Lin L, Li Q, Sun C, Li Y: Multigenerational effects of parental prenatal exposure to famine on adult offspring cognitive function. Scientific reports 2015, 5:13792.

80. He S, Li J, Wang Z, Wang L, Liu L, Sun X, Shohaib SA, Koenig HG: Early-life exposure to famine and late-life depression: Does leukocyte telomere length mediate the association? Journal of affective disorders 2020, 274:223-228.

81. Stein AD, Pierik FH, Verrips GH, Susser ES, Lumey LH: Maternal exposure to the Dutch famine before conception and during pregnancy: quality of life and depressive symptoms in adult offspring. Epidemiology (Cambridge, Mass) 2009, 20(6):909-915. 